\title{
Conocimiento básico de los riesgos del uso de analgésicos no opioides en pacientes ambulatorios
}

\author{
Svetlana Vladislavovna Doubova (Dubova), MD, MSc, (1) Dolores Mino-León, MD, MSc, ${ }^{(2)}$ \\ Laura del Pilar Torres-Arreola, MD, MSc, (1) Guillermina Romero-Quechol, ESP(I)
}

\author{
Doubova (Dubova) SV, Mino-León D, \\ Torres-Arreola LP, Romero-Quechol G. \\ Conocimiento básico de los riesgos \\ del uso de analgésicos no opioides \\ en pacientes ambulatorios. \\ Salud Publica Mex 2007;49:429-436.
}

\section{Resumen}

Objetivo. Evaluar el conocimiento básico de los pacientes acerca de los analgésicos no opioides (ANOP) e identificar los posibles factores relacionados con la falta de información sobre este tipo de analgésicos. Material y métodos. Participaron 629 pacientes $\geq 50$ años con síndrome doloroso de origen no oncológico y que recibieron ANOP. Se analizaron sus características generales, la información recibida y su conocimiento sobre ANOP. La variable dependiente fue la falta de conocimiento básico (FCB) sobre ANOP. Se realizó análisis descriptivo y bivariado. Resultados. Del total de participantes, $64.2 \%$ tuvo FCB; $28 \%$ desconocía la forma correcta de tomar ANOP y $48 \%$ sabía que ocasionan trastornos gastrointestinales. Factores asociados con la FCB: no recibir información sobre ANOP ( $R M=2.22$; IC95\% I.32$3.70)$, escolaridad $<7$ años ( $R M=1.87 ;$ IC95\% I.33-2.63) y duración del dolor < 4 años (RM=I.70; IC95\% I.22-2.37). Conclusiones. Los pacientes carecen de conocimiento y reciben poca información acerca de ANOP. Es indispensable promover acciones para solucionar este problema.

Palabras clave: conocimiento; analgésicos no opioides; pacientes ambulatorios; México
Doubova (Dubova) SV, Mino-León D,

Torres-Arreola LP, Romero-Quechol G.

Basic knowledge of risks

for non-opioid analgesics (NOA)

in ambulatory patients.

Salud Publica Mex 2007;49:429-436.

\begin{abstract}
Objective. To describe patients' knowledge of non-opioid analgesics (NOA) and to identify factors associated with patients' lack of basic knowledge (LBN) on this type of medication. Material and Methods. A total of 629 ambulatory patients older than 50 years of age, with non-malignant pain syndrome, attended to two family medicine clinics and received seven day prescriptions for NOA. The data on patients' general characteristics, the information they received and their actual knowledge of NOA were analyzed using descriptive statistics and bivariate analysis. Results. A total of $64.2 \%$ had LBN; $28 \%$ did not know how to take NOA properly, and $48 \% \mathrm{knew}$ that these drugs cause gastrointestinal adverse effects. The factors significantly associated with LBN on NOA included: failure to receive information on NOA (OR:2.22,

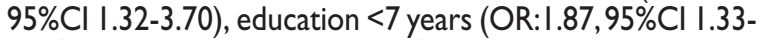
2.63 ) and pain duration $<4$ years (OR:I.70,95\% Cl I.22-2.37). Conclusion. Patients lack knowledge and receive little information on NOA. It is important to encourage actions to tackle this problem.
\end{abstract}

Key words: non-opioid analgesics; knowledge; ambulatory patients; Mexico

(I) Unidad de Investigación Epidemiológica y en Servicios de Salud. Centro Médico Nacional Siglo XXI. Instituto Mexicano del Seguro Social (IMSS). México DF.

(2) Coordinación en Investigación en Salud. IMSS. México DF.

Fecha de recibido: 28 de marzo de 2007 - Fecha de aceptado: II de septiembre de 2007

Solicitud de sobretiros: Dra. Svetlana Vladislavovna Doubova (Dubova). Unidad de Investigación Epidemiológica y en Servicios de Salud. Centro Médico Nacional Siglo XXI. Instituto Mexicano del Seguro Social. Av. Cuauhtémoc 330, Col Doctores, CP 06725, México, DF.

Correo electrónico: svetlana.doubova@imss.gob.mx 
L a falta de información de los usuarios y prescriptores en lo referente al uso de los medicamentos y de sus potenciales consecuencias constituye un problema creciente; el ingreso hospitalario asociado con el uso inapropiado de medicamentos ocurre frecuentemente y es un indicador importante. ${ }^{1,2} \mathrm{~A}$ lo anterior se suma el hecho de que de 60 a 70\% de los casos que presentan efectos adversos a medicamentos (EAM) son prevenibles a través de una prescripción apropiada. ${ }^{3,4}$

El personal de salud debe proporcionar información y educar a los usuarios sobre la utilización de los medicamentos, situación que desafortunadamente no ocurre. ${ }^{5-7}$ La falta de información se traduce en dificultad para identificar los efectos adversos, en menor cumplimiento y en una utilización errónea de las medicinas, todo lo cual posiblemente deriva en una mayor utilización de servicios de salud. Algunos estudios han revelado que los pacientes perciben la necesidad de contar con mayor información acerca de los medicamentos que se les prescriben, así como de sus repercusiones en la salud. ${ }^{8-10}$

La Organización Mundial de la Salud identifica como una prioridad la educación a la población por los profesionales de salud en el uso racional de los medicamentos. En ese sentido, las tres metas principales son: a) mejorar el conocimiento sobre los medicamentos -qué son y cuál es su papel-;b) mejorar el conocimiento sobre el beneficio y riesgo de los medicamentos, y c) motivar a los pacientes y a sus familias en el proceso de la decisión-informada. ${ }^{11}$ Cuando el personal de la salud proporciona suficiente información al paciente, aumentan en este último la confianza y la adherencia terapéutica y disminuye la frecuencia de EAM prevenibles. ${ }^{12-14}$

Se ha notificado que en el grupo terapéutico de los analgésicos no opioides (ANOP), los antiinflamatorios no esteroideos (AINEs) son medicamentos causantes de frecuentes EAM prevenibles ${ }^{4}$ y que tanto los médicos como los pacientes carecen de conocimiento suficiente para utilizarlos. ${ }^{15-17}$ Un estudio realizado en Inglaterra indicó que 34\% de los pacientes con osteoartrosis no sabían cómo utilizar los AINEs. ${ }^{17}$

Actualmente en México, y en particular en el Instituto Mexicano del Seguro Social (IMSS), sistema de seguridad social que atiende a población empleada en el sector formal y que representa $40 \%$ de la población del país, se otorgan un número importante de consultas a pacientes que requieren ANOP. En 2006, 10.7\% ( $n=1665$ 970) del total nacional de las consultas de primera vez otorgadas en medicina familiar $(n=15621$ 362) en el IMSS, correspondieron a problemas osteomusculares y traumatismos. ${ }^{18}$ En órdenes de magnitud esto representa una importante cifra de pacientes que deben recibir la información y que, potencialmente, pueden tener EAM por utilización inadecuada de los medicamentos.

La literatura acerca del tema indica que en México se carece de información relacionada con el conocimiento de los pacientes acerca de los ANOP y de sus posibles efectos adversos.

En 2006 se inició una investigación con el propósito de evaluar el impacto de una intervención educativa en médicos y pacientes sobre el uso de los ANOP; a partir de dicha investigación, se estableció el objetivo del presente trabajo: evaluar el conocimiento básico de los pacientes acerca de la utilización de los ANOP (AINEs y paracetamol), e identificar posibles factores relacionados con la falta de este conocimiento.

\section{Material y métodos}

El presente estudio es un análisis secundario de datos del estudio EEA, ${ }^{*}$ realizado en dos unidades de medicina familiar (UMF) del IMSS en la Ciudad de México, durante 2006. Se analizó la información de 629 pacientes ambulatorios mayores de 50 años, que acudieron a consulta con 127 médicos familiares por síndrome doloroso de origen no oncológico. La información se obtuvo después de que el paciente salió de consulta y recibió la prescripción de ANOP por un periodo mayor a siete días.

A los pacientes se les aplicó un cuestionario integrado por preguntas con opciones múltiples (tres primeras preguntas) y abiertas (preguntas 4-8) que permitió evaluar el conocimiento sobre la utilización y los riesgos del consumo de estos fármacos, a través de ocho preguntas: 1) Manera de tomar los ANOP; 2) Efectos adversos que pueden ocasionar; 3) Acciones que debe realizar el paciente en caso de presentar efectos secundarios a los ANOP; 4) Personas que están en mayor riesgo de desarrollar efectos secundarios a los ANOP; 5) ANOP que provocan menos daño; 6) Acciones que debe realizar el paciente cuando se le olvida tomar una dosis de ANOP; 7) Acciones que se deben realizar en caso de tomar por equivocación una dosis mayor a la prescrita, y 8) Conducta del paciente cuando presenta dolor severo y no tiene analgésicos en su casa.

Se consideró como variable dependiente la falta de conocimiento básico (FCB) sobre los ANOP, misma que se definió como la afirmación del paciente de no tener

\footnotetext{
* Estudio de la Estrategia Educativa dirigida a los médicos y a los pacientes mayores de 50 años sobre la utilización de los analgésicos no opioides en el primer nivel de atención.
} 
conocimiento con respecto a, o bien como la respuesta incorrecta del paciente en al menos una de las tres primeras preguntas del cuestionario, dado que éstas cubren los aspectos mínimos que deben conocerse para prevenir, reconocer y tomar acciones inmediatas.

Como conocimiento básico se consideraron las respuestas correctas del paciente a las tres primeras preguntas, indicando para la primera que la mejor manera de tomar los ANOP es junto con los alimentos y/o leche, o inmediatamente después de los alimentos; en el caso de la segunda pregunta, cuando mencionó, por lo menos, los efectos gastrointestinales entre las molestias que los ANOP pueden ocasionar y, para la tercera, cuando contestó que debía ir inmediatamente a consulta con su médico familiar y/o suspender el medicamento en caso de presentar efectos secundarios. Las otras preguntas del cuestionario fueron consideradas como complementarias.

Otros aspectos que cubrió el cuestionario fueron: características generales de los pacientes (sexo, edad, estado civil, escolaridad y la ocupación que se evaluó al preguntar al paciente si trabajaba o no fuera del hogar); antecedentes personales patológicos; características del dolor (origen, duración e intensidad, la cual se midió con una escala análoga visual de 0-10 cm); automedicación de analgésicos, e información recibida sobre los ANOP por el personal de salud (médico familiar, médico particular, enfermera de UMF).

El cuestionario fue diseñado especialmente para el estudio, con base en las recomendaciones de la OMS y su aplicabilidad se evaluó con un estudio piloto en una UMF que no fue incluida en el estudio original. La entrevista se aplicó en dos UMF seleccionadas de manera convencional. Dos enfermeras previamente capacitadas acudieron a las UMF para entrevistar a los pacientes que aceptaron participar en el estudio, tomando en forma consecutiva 4 a 5 pacientes por médico y cubriendo todos los médicos en ambos turnos. La tasa de aceptación fue de $97 \%$; todos los pacientes entrevistados contestaron completamente a la encuesta.

El Comité de Ética de la Comisión Nacional del IMSS evaluó y aceptó el proyecto principal.

\section{Análisis estadístico}

Se realizó análisis estadístico descriptivo con medidas de tendencia central para variables continuas, y frecuencias relativas y absolutas para variables categóricas.

El análisis bivariado permitió evaluar diferencias entre el grupo de pacientes con FCB y aquel con conocimiento básico (CCB). Se utilizó prueba de ji cuadrada para variables categóricas, se obtuvieron las razones de momios crudas con sus intervalos de confianza al 95\%.(IC 95\%) y se definió una $p<0.05$ como estadísticamente significativa.

El análisis se llevó a cabo mediante el paquete estadístico Stata 8.0 (Stata 8.0; Stata Corp; College Station, Tx). El estudio tuvo un poder de $99 \%$ para detectar una diferencia de $10 \%$ respecto a los pacientes que recibieron información sobre ANOP entre los grupos (pacientes CCB y FCB) con un alfa de 0.05 .

\section{Resultados}

Se incluyó a 629 pacientes, con edades entre 50 y 94 años y una mediana de 69 años de edad; $78.2 \%$ pertenecía al sexo femenino, $82.5 \%$ se dedicaba al hogar o estaban jubilados y más de la mitad $(51.2 \%)$ no tenía pareja. La mediana de la escolaridad fue de seis años.

Cerca de 90\% de la población entrevistada acudió a consulta con el médico familiar por dolor músculoesquelético, que en más de $60 \%$ de los casos limitaba su actividad normal (cuadro I). La intensidad promedio del dolor fue de $6.23 \pm 2.2$ en la escala análoga visual y su duración en años de $6.26 \pm 5.3$.

Todos los pacientes recibieron al menos una prescripción de ANOP, del cuadro básico de medicamentos del IMSS. El paracetamol se prescribió en $36.6 \%$ de los casos y diferentes tipos de antiinflamatorios no esteroideos (AINEs) 89.7 por ciento. De los pacientes, $8.1 \%$ recibió una prescripción con dos AINEs que con mayor frecuencia fueron diclofenaco, naproxeno, piroxicam, indometacina y celecoxib. De los pacientes que tomaron paracetamol (37\%) sólo $10.3 \%$ lo hizo como único analgésico del grupo ANOP; el resto lo ingería con otros ANOP. Además, todos los pacientes que tomaron paracetamol informaron haber tomado anteriormente (en el presente año) alguno de los AINEs.

En relación con la información que recibieron sobre la utilización de este tipo de fármacos, únicamente $7 \%$ de los pacientes comentó que durante el último año recibió algún tipo de información verbal por parte del médico familiar y que la misma se relacionó con la dosis y los posibles efectos adversos que estos fármacos pueden ocasionar (cuadro II).

Alrededor de 30\% (117) de los pacientes desconocía que los analgésicos se deben tomar con los alimentos o con leche y únicamente $48 \%$ sabía que los analgésicos pueden ocasionar algún daño al sistema digestivo.

Del total de los pacientes, 27\% contestó que no sabían qué hacer en caso de que se presentaran molestias secundarias a los ANOP; $42 \%$ respondió que era necesario acudir inmediatamente a consulta con el médico familiar, y 16\% dijo que había que suspender el 


\section{Cuadro I}

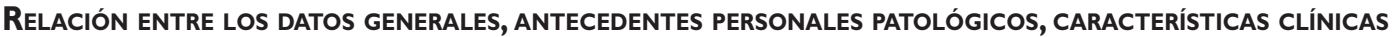
DEL DOLOR, AUTOMEDICACIÓN Y CONOCIMIENTO BÁSICO SOBRE LOS ANALGÉSICOS NO OPIOIDES DE LA POBLACIÓN DE ESTUDIO. Dos UMF del IMSS México DF, 2006

$\begin{array}{cccccc} & \text { Total } & C C B & F C B & \text { Razón de momios } & \text { cruda } \\ \text { Variables } & n=629 & n=225 & n=404 & \text { IC 95\% }\end{array}$

I. Datos generales

Sexo:

\begin{tabular}{crrrrr} 
Femenino & $492(78.2)$ & $183(81.3)$ & $309(76.5)$ & 1.0 & \\
\hline Masculino & $137(21.8)$ & $42(18.7)$ & $95(23.5)$ & 1.34 & $0.89-2.01$ \\
& & & & & \\
Vive con pareja & $307(48.8)$ & $116(51.6)$ & $191(47.3)$ & 1.0 & \\
\hline Vive sin pareja (soltero, viudo, divorciado) & $322(51.2)$ & $109(48.4)$ & $213(52.7)$ & 1.18 & $0.85-1.64$
\end{tabular}

Ocupación:

\begin{tabular}{|c|c|c|c|c|c|}
\hline No trabaja & $519(82.5)$ & $193(85.8)$ & $326(80.7)$ & 1.0 & \\
\hline Trabaja fuera del hogar & $110(17.5)$ & $32(14.2)$ & $78(19.3)$ & 1.44 & $0.92-2.26$ \\
\hline Edad $<70$ años & $319(50.7)$ & $110(48.9)$ & $209(51.7)$ & 1.0 & \\
\hline Edad $\geq 70$ años & $310(49.3)$ & $115(5 I .1)$ & $195(48.3)$ & 0.89 & $0.64-1.24$ \\
\hline Escolaridad $\geq 7$ años & $216(34.3)$ & $98(43.6)$ & $118(29.2)$ & 1.0 & \\
\hline Escolaridad $<7$ años* & $413(65.7)$ & $127(56.4)$ & $286(70.8)$ & $1.87^{*}$ & $1.33-2.63$ \\
\hline
\end{tabular}

II. Antecedentes patológicos

Enfermedades crónicas $\leq 3$

$255(56.4)$

$274(43.6)$

\begin{tabular}{ll}
$122(54.2)$ & $233(57.7)$ \\
\hline $103(45.8)$ & $17 \mid(42.3)$
\end{tabular}

1.0

Enfermedades crónicas $\geq 4$

$274(43.6) \quad 103(45.8) \quad$ |7| (42.3)

1.0

Características clínicas del dolor

Dolor neuropático o psicógeno

Dolor músculo-esquelético

$17(2.7)$

$602(95.7)$

$6(2.7)$

II (2.7)

1.0
1.02

$0.37-2.80$

Intensidad del dolor $\geq 5.5$ puntos en EAV

$314(49.9) \quad 124(55.1)$

Intensidad del dolor $<5.5$ puntos en EAV

$315(50$.

I0I (44.9) $214(53.0)$

1.0

Duración del dolor $\geq 4$ años

$324(51.5)$

$305(48.5)$

$135(60.0)$

$189(46.8)$

$(6.8)$

1.0
$1.70^{*}$

$0.99-1.92$

Dolor no restringió la actividad del paciente

$223(35.5)$

$85(37.8) \quad 138(34.2)$

$(65.8)$

1.0
1.17

$1.22-2.37$

Dolor restringió la actividad normal del paciente $406(64.6)$

140 (62.2) $266(65.8)$

$0.83-1.64$

IV. Automedicación de analgésicos durante el último año

\begin{tabular}{lrrrrr} 
No & $516(82.0)$ & $185(82.2)$ & $331(81.9)$ & 1.0 & \\
\hline Sí & $113(18.0)$ & $40(17.8)$ & $73(18.1)$ & 1.02 & $0.66-1.56$
\end{tabular}

V. Recibieron información sobre los ANOP durante el último año por personal del salud*

\begin{tabular}{lrrrrr} 
Sí & $66(10.5)$ & $35(15.6)$ & $31(7.7)$ & 1.0 & \\
\hline No & $563(89.5)$ & $190(84.4)$ & $373(92.3)$ & $2.22^{*}$ & $1.32-3.70$
\end{tabular}

$* p<0.05$

$\mathrm{CCB}=$ con conocimiento básico sobre los analgésicos no opioides

$\mathrm{FCB}=$ falta de conocimiento básico

$E A V=$ escala análoga visual de $0.0-10$ 


\section{Cuadro II \\ TIPO DE INFORMACIÓN VERBAL QUE RECIBIERON LOS PACIENTES DURANTE EL ÚLTIMO AÑO ACERCA DEL USO DE ANOP. Dos UMF deL IMSS Méxıco DF, 2006}

\begin{tabular}{|c|c|}
\hline & $\begin{array}{c}n=629 \\
n(\%)\end{array}$ \\
\hline Información relacionada con la prescripción & $66(10.5)$ \\
\hline Duración del tratamiento & $12(1.9)$ \\
\hline Dosis & $42(6.7)$ \\
\hline Intervalo de administración & $35(5.6)$ \\
\hline Manera de tomar los analgésicos & $34(5.4)$ \\
\hline Efectos adversos & $39(6.2)$ \\
\hline \multicolumn{2}{|l|}{ Acciones en caso de presentar molestias } \\
\hline secundarias al uso de ANOP & $20(3.2)$ \\
\hline \multicolumn{2}{|c|}{ Personal que informó al paciente sobre los analgésicos } \\
\hline \multicolumn{2}{|l|}{ no opioides } \\
\hline Médico familiar & $44(6.9)$ \\
\hline Otro (médico particular, especialista, etc.) & $22(3.5)$ \\
\hline
\end{tabular}

medicamento. Por otro lado, $57.1 \%$ ignoraba qué tipo de pacientes tienen un riesgo más elevado de presentar EAM por ANOP y de los que refirieron conocer esta información (42.9\%), 25.4\% contestó que eran los adultos mayores, $15.1 \%$ mencionó a los niños y $5.9 \%$ a los pacientes con enfermedades gastrointestinales crónicas.

Los pacientes evaluaron al paracetamol, diclofenaco y a la aspirina como menos "dañinos".

Ante la pregunta de qué hacer si se les olvida una dosis, $76.5 \%$ de los pacientes contestó que no la repondrían, $16.9 \%$ que no sabría qué hacer y $6.7 \%$ que la tomaría al momento de recordarlo.

Con respecto a la pregunta de qué hacer en caso de tomar una dosis de ANOP mayor a la prescrita, 54.1\% respondió que no sabrían qué hacer; $17 \%$ que acudiría con el médico y $6.8 \%$ que se realizaría un lavado de estómago o se provocarían el vómito.

Ante el planteamiento de qué harían si se presentara dolor intenso y no contaran con un analgésico en casa, $36.9 \%$ respondió que soportarían el dolor; $32 \%$ que acudiría al médico y tan sólo $12.6 \%$ que se aplicaría calor local (cuadro III).

La FCB se encontró en $64.2 \%$ de los casos y el no recibir información sobre los ANOP por parte del personal de la salud se relacionó con esta FCB ( $R M=2.22$; IC95\% 1.32 -3.70). Además, los factores relacionados con FCB fueron la educación $<7$ años $(R M=1.87$; IC95\%
1.33 -2.63) y la duración del dolor $<4$ años $(R M=1.70$; IC95\% 1.22 -2.37) (cuadro I).

\section{Discusión}

Se ha reconocido que el desconocimiento de los pacientes sobre el uso de los medicamentos es un problema frecuente e importante y que puede repercutir en su estado de salud. Es relevante que los pacientes conozcan la forma correcta de utilizar los ANOP, debido a su elevado consumo y a la frecuencia de reacciones adversas. ${ }^{19,20}$

En este estudio se informó que $28.1 \%$ de los pacientes ignoraba la forma correcta de tomar los ANOP; lo cual concuerda con los hallazgos de diversos investigadores como Hill y colaboradores, ${ }^{17}$ quienes informaron que $34 \%$ de los pacientes con osteoartritis desconocían la forma correcta de tomar los AINEs; Ridout y colaboradores, ${ }^{9}$ notificaron que más de $72 \%$ de la población estudiada (pacientes ambulatorios de la comunidad de Southampton, Inglaterra) ignoraba los posibles efectos adversos de los medicamentos que estaba tomando; Ascione y colaboradores, ${ }^{21}$ encontraron que sólo $10 \%$ de los pacientes con enfermedades cardiovasculares sabía de los efectos secundarios negativos de los medicamentos que consumían y Busson y colaboradores ${ }^{5}$ detectaron que $43 \%$ de los pacientes que estudiaron no sabía qué hacer en caso de presentarse EAM.

En el presente estudio, $24 \%$ de los pacientes desconocía las acciones que deben realizarse en caso de olvidar tomar una dosis de analgésicos y más de $70 \%$ tampoco sabía qué hacer en caso de ingerir accidentalmente una dosis mayor a la prescrita por el médico. Indudablemente, la falta de conocimiento sobre estos aspectos favorece un mayor riesgo de padecer los efectos adversos de los medicamentos.

Los pacientes carecen de conocimiento no sólo sobre los riesgos que ocasiona el consumo de analgésicos, sino también acerca de los métodos no farmacológicos para combatir el dolor: únicamente $21.4 \%$ de los pacientes conocía alguno de los métodos no farmacológicos. Este hallazgo contrasta con un estudio realizado en Estados Unidos donde se notificó que 68\% de los pacientes entrevistados refirió conocer y usar los métodos no farmacológicos para combatir el dolor. ${ }^{19}$

El escaso conocimiento de los pacientes evaluados en este estudio acerca del uso de los ANOP, se puede explicar por la ausencia de información de parte del personal de salud y, en particular, de su médico familiar. Este dato es preocupante pues resultó inferior a lo notificado en otros estudios donde de 56 a $62 \%$ de los médicos informa a los pacientes sobre la dosis que deben tomar; $75 \%$ lo hacen con respecto a la duración 


\section{Cuadro III \\ Conocimiento sobre LA UTILIZACIÓN Y LOS RIESGOS DEL CONSUMO de ANOP. Dos UMF DEL IMSS MÉxico DF, 2006}

\begin{tabular}{|c|c|}
\hline \multirow{2}{*}{\multicolumn{2}{|c|}{$\begin{array}{l}\text { Aspectos relacionados } \\
\text { I. Manera de tomar los analgésicos }\end{array}$}} \\
\hline & \\
\hline Correcta & $512(7 \mid .9)$ \\
\hline 2. Conocimiento sobre los efectos adversos que pueden & \\
\hline ser ocasionados por los ANOP a nivel & $304(48.3)$ \\
\hline Gastrointestinal & $279(44.4)$ \\
\hline Cardiovascular & $8(1.3)$ \\
\hline Renal & $25(3.9)$ \\
\hline Hepático & $12(1.9)$ \\
\hline Alérgicos & $21(3.3)$ \\
\hline Otros & $12(1.9)$ \\
\hline
\end{tabular}

3. Acciones que realizaría en caso de presentar efectos adversos a los ANOP

No sabe

I7I (27.2)

Ir inmediatamente a consulta con su médico $265(42.1)$ Suspender el ANOP $99(15.7)$

Tomar otro medicamento $30(4.8)$ Disminuir la dosis del ANOP $4(0.6)$

Tomar el ANOP con algún alimento o leche

$60(9.5)$

4. Conocimiento de qué personas tienen mayor riesgo de presentar efectos secundarios a los ANOP*

\begin{tabular}{lr} 
Lo ignora & $270(42.9)$ \\
\hline Personas de la tercera edad & $160(25.4)$ \\
\hline Niños & $95(15.1)$ \\
\hline Personas con enfermedades gastrointestinales & $37(5.9)$ \\
\hline Pacientes diabéticos & $33(5.3)$ \\
\hline Personas con enfermedades cardiovasculares & $28(4.4)$ \\
\hline $\begin{array}{l}\text { Personas que toman varios analgésicos al mismo } \\
\text { tiempo o los toman por periodos largos }\end{array}$ & $23(3.7)$ \\
\hline Personas que se automedican & $13(2.1)$ \\
\hline Personas alérgicas & $\mathrm{II}(\mathrm{I} .8)$ \\
\hline Personas que toman bebidas alcohólicas y/o fuman & $10(1.6)$ \\
\hline Otros & $12(1.9)$
\end{tabular}

Aspectos relacionados

5. Conocimiento acerca de qué tipo de ANOP provoca menos efectos adversos ${ }^{\ddagger}$

\begin{tabular}{lr} 
No conoce & $444(70.6)$ \\
\hline Aspirina & $32(5.0)$ \\
\hline Naproxeno & $12(1.9)$ \\
\hline Piroxicam & $12(1.9)$ \\
\hline Indometacina & $8(1.3)$ \\
\hline Paracetamol & $87(13.9)$ \\
\hline Diclofenaco & $45(7.2)$ \\
\hline Otros & $7(1.3)$
\end{tabular}

6. Qué debería hacer si se le olvida tomar una dosis del ANOP

No sabe

$106(16.9)$

Tomárselo inmediatamente cuando se acuerda

$42(6.7)$

Esperar al próximo periodo de dosificación

$48 \mathrm{I}(76.5)$

7. Qué debería hacer en caso de tomar accidentalmente una dosis mayor a la indicada por el médico

No sabe $340(54.1)$

Tomar algún lácteo (leche, yogurt etc) $85(13.5)$

Tomar suficiente agua o té $24(3.8)$

Comer algo $8(1.3)$

Dejaría pasar más tiempo para tomarse la siguiente dosis $13(2.9)$

Acudir al médico

Provocar el vómito o hacer lavado del estómago $\quad 43(6.8)$

Tomar carbonato para contrarrestar el efecto del ANOP $4(0.6)$

8. Qué haría si presenta dolor severo y no tiene analgésicos en casa

No sabe

$232(36.9)$

Acude al médico $201(32.0)$

Compra algún analgésico

Se aplica calor local $79(12.6)$

Toma té caliente $33(5.2)$

Realiza ejercicio físico de pies y manos $8(1.3)$ Se da un masaje con cepillo o cojín electrónico $4(0.6)$ Se pone hielo $4(0.6)$ 
del tratamiento; de 23 a $82 \%$ lo hacen con respecto a los posibles efectos adversos, y de 26 a $34 \%$ informa acerca de la manera en que deben tomarse los medicamentos. ${ }^{22,23}$ Sin embargo, se encontró que en el grupo de los pacientes que recibieron información, 56\% tenía conocimiento básico sobre estos medicamentos. Además, una menor escolaridad del paciente y una menor duración del dolor se relacionaron con la ausencia del conocimiento sobre los ANOP, lo que hace suponer que los pacientes con mayor escolaridad y un tiempo más prolongado de experimentar el dolor, buscan otras fuentes de información sobre los analgésicos.

Con el fin de promover el uso racional e inocuo de los medicamentos, en varios países se ha propuesto incluir información dirigida al paciente, dentro del paquete del medicamento. ${ }^{24,25}$ Se recomendó que dicha información fuese imparcial, suficiente y, al mismo tiempo, breve, escrita en un lenguaje comprensible y lo menos técnico posible. El mensaje debe contener datos para el uso correcto del medicamento y acerca de sus posibles efectos adversos. Esta información tendría que ser adicional a la ficha técnica que se coloca habitualmente y que es un documento científico-técnico destinado a informar al personal de salud respecto del medicamento. Se ha encontrado que a pesar de que los pacientes valoran altamente la información impresa acerca de los medicamentos, no la consideran como un sustituto de la información proporcionada por su médico tratante y que ambas fuentes son necesarias y complementarias. ${ }^{26}$

Una de las limitaciones de este estudio es que sólo permite una aproximación al problema de la falta de conocimiento de los pacientes sobre ANOP, lo cual podría atribuirse a que se trata de un análisis secundario de datos y posiblemente a que existen otros factores relacionados, pero que no fueron considerados en esta investigación. Así, por ejemplo, la calidad de la comunicación para informar a los pacientes acerca de los ANOP y en qué condiciones se otorga ésta. Por otra parte, el estudio no permitió conocer si el paciente estaba satisfecho con la información.

Desde el punto de vista del análisis estadístico, se considera que el análisis bivariado aporta información plausible para identificar en una primera aproximación los factores relacionados a la falta de conocimiento sobre ANOP; empero, es indispensable diseñar estudios ad hoc para obtener información más robusta en estos aspectos.

Es posible concluir que los pacientes estudiados carecen de conocimiento y reciben poca información sobre la correcta utilización y riesgos que ocasionan los ANOP; por lo que es importante destacar, entre los profesionales de la salud (médicos, enfermeras, farma- céuticos), la importancia clínica que reviste el hecho de brindar información suficiente y de calidad a los pacientes que requieren este tipo de fármacos.

\section{Referencias}

I.Winterstein AG, Sauer BC, Hepler CD, Poole C. Preventable drugrelated hospital admissions. Ann Pharmacother 2002;36: I238-I 248. 2. Howard RL, Avery AJ, Slavenburg S, Royal S, Pipe G, Lucassen P, et al. Which drugs cause preventable admissions to hospital? A systematic review. Br J Clin Pharmacol 2007;63:I36-I47.

3. Pirmohamed M, James S, Meakin S, Green C, Scout AK, Walley T], et al. Adverse drug reactions as a cause of admission to hospital: prospective analysis of 18820 patients. BMJ 2004;329:15-19.

4. Gurwitz JH, Field TS, Harrold LR, Rothschild J, Debellis K, Seger AC, et al. Incidence and preventability of adverse drug events among older persons in the ambulatory setting. JAMA 2003;289: I I07- I I I6.

5. Busson M, Dunn APM. Patients knowledge about prescribed medicines. Pharmaceutical Journal 1986;236:624-626.

6. O'Connell MB, Jonson JF. Evaluation of medication knowledge in elderly patients. Ann Pharmacother 1992;26:919-921.

7. Enlund $\mathrm{H}$,Vainio K,Wallenius S, Poston JW.Adverse drug effects and the need for drug information. Med Care 1991;29:558-564.

8. Laine C, Davidoff F, Lewis CE, Nelson EC, Nelson E, Kessler RC, et al. Important elements of outpatient care: a comparison of patients and physicians opinions. Ann Intern Med 1996;125:640-645.

9. Ridout S, Waters WE, George CF. Knowledge of and attitudes to medicines in the Southampton community. Br J Clin Pharmacol 1086;2I:701-7|2.

10. Gibbs S,Waters WE, George CF. Communicating information to patients about medicine.J R Soc Med 1990;83;292-297.

I I.WHO:EDM- I8. Public education in drug use: a growing need. Disponible en: http://mednet2. who.int/edmonitor/edition/edm I8a.html 12. De Young M. Research on the effects of pharmacist-patient communication in institutions and ambulatory care sites, 1969-1994.Am J Health Syst Pharm 1996;53:1277-129I.

I3. Tang PC, Newcomb C. Informing patients: a guide for providing patient health information. J Am Med Inform Assoc 1998; 5:563-570.

14. O'Neil CK, Poirer TI. Impact of patient knowledge, patientpharmacist relationship, and drug perceptions on adverse drug therapy outcomes. Pharmacotherapy 1998;18:333-340.

I5. Keys J, Beardon PH, Lau CC, McDevitt DG. General practitioners use of mom-steroidal anti-inflammatory drugs in Tayside and fife regions. J $R$ Soc Med I992; I 1 7:684-689.

16. Tamblyn R, Berkson L, Dauphinee WD, Gayton D, Grad R, Huang A, et al. Unnecessary prescribing of NSAIDs and the management of NSAID-related gastropathy in medical practice. Ann Intern Med 1997; 127:429-438.

17. Hill J, Bird H. Patient knowledge and misconceptions of osteoarthritis assessed by a validated self-completed knowledge questionnaire (PKQ-OA). Rheumatology 2007;46:796-800.

18. Instituto Mexicano del Seguro Social. Información estadística en salud. Consultas de Medicina Familiar. Primera Vez 2006. Disponible en: http://www.imss.gob.mx/dpm/dties/Celula.aspx?ID=SC06_00_00_ 01_06\&OPC=opc07\&SRV=A2006

19. Vallerand AH, Fouladbakhsh J, Templin T. Patients choices for the selftreatment of pain. Appl Nurs Res 2005; 18:90-96.

20. WHO Collaborating Centre for International drug monitoring.

Viewpoint part I. Uppsala, Sweden:The Uppsala Monitoring Centre,2003. 
21. Ascione FJ, Kirscht JP, Shimp LA.An assessment of different components of patient medication knowledge. Med Care 1986;24:1018-1028.

22. McMahon T, Clark CM, Bailie GR. Who provides patients with drug information ? BMJ 1987;294:355-356.

23. Morris LA, Grossman R, Barkdol GL, Gordon E, Soviero C.A survey of patients sources of prescription drug information. AJPH I984;74: II6I-II62.

24. Food and Drug Administration--Prescription drug products; patient package inserts requirements. Final rule. Fed Regist 1980;45( I79 Pt 2):60754-60784.
25. Rubio Terrés C. La regulación de los medicamentos en España. Med Clin 1992;98:741-748.

26. Grime J, Blenkinsopp A, Raynor DK, Pollock K, Knapp P.The role and value of written information for patients about individual medicines: a systematic review. Health Expect 2007; 10:286-298. 